\title{
naked cuticle targets dishevelled to antagonize Wnt signal transduction
}

\author{
Raphaël Rousset, ${ }^{1,2,7}$ Judith A. Mack, ${ }^{1,2,5,7}$ Keith A. Wharton, Jr., ${ }^{1-3,6}$ Jeffrey D. Axelrod, $^{3}$ \\ Ken M. Cadigan, ${ }^{1,4}$ Matthew P. Fish, ${ }^{1,2}$ Roel Nusse, ${ }^{1}$ and Matthew P. $\operatorname{Scott}^{1,2,8}$ \\ Departments of ${ }^{1}$ Developmental Biology, ${ }^{2}$ Genetics, and ${ }^{3}$ Pathology, Howard Hughes Medical Institute, Beckman Center B300, \\ Stanford University School of Medicine, Stanford, California 94305, USA; ${ }^{4}$ Department of Biology, Kraus Natural Science \\ Building, University of Michigan, Ann Arbor, Michigan 48109, USA
}

In Drosophila embryos the protein Naked cuticle (Nkd) limits the effects of the Wnt signal Wingless (Wg) during early segmentation. nkd loss of function results in segment polarity defects and embryonic death, but how nkd affects Wnt signaling is unknown. Using ectopic expression, we find that Nkd affects, in a cell-autonomous manner, a transduction step between the Wnt signaling components Dishevelled (Dsh) and Zeste-white 3 kinase (Zw3). Zw3 is essential for repressing Wg target-gene transcription in the absence of a $\mathrm{Wg}$ signal, and the role of $\mathrm{Wg}$ is to relieve this inhibition. Our double-mutant analysis shows that, in contrast to Zw3, Nkd acts when the Wg pathway is active to restrain signal transduction. Yeast two hybrid and in vitro experiments indicate that Nkd directly binds to the basic-PDZ region of Dsh. Specially timed Nkd overexpression is capable of abolishing Dsh function in a distinct signaling pathway that controls planar-cell polarity. Our results suggest that Nkd acts directly through Dsh to limit Wg activity and thus determines how efficiently Wnt signals stabilize Armadillo (Arm)/ $\beta$-catenin and activate downstream genes.

[Key Words: Wnt/wingless; naked cuticle; dishevelled; zeste-white 3; segmentation; Drosophila]

Received November 27, 2000; revised version accepted January 22, 2001.

Secreted Wnt proteins act as potent mitogens and cellfate regulators in organisms ranging from nematodes to humans. In vertebrates they specify cell fate and control growth in a variety of developmental processes, including brain development, limb formation, axis specification, and gastrulation (for review, see Cadigan and Nusse 1997). In the fruit fly Drosophila, the Wnt protein Wingless $(\mathrm{Wg})$ establishes segment polarity during embryogenesis and is involved in multiple additional patterning events throughout later development (Cadigan and Nusse 1997). wg is first expressed in the developing epidermis in stripes just anterior to cells expressing the engrailed (en) gene and is necessary to maintain en transcription (DiNardo et al. 1988; Martinez Arias et al. 1988). hedgehog (hh) is expressed in the en-expressing cells and positively regulates $w g$ expression in the anterior cells (Ingham et al. 1991; Lee et al. 1992). This positive-feedback loop establishes parasegmental bound-

Present addresses: ${ }^{5}$ Cleveland Clinic Florida, Research Laboratory, Building 2950, 3000 West Cypress Creek Road, Ft. Lauderdale, FL 33309, USA; ${ }^{6}$ Departments of Pathology and Molecular Biology, University of Texas Southwestern Medical Center NB6.440, 5323 Harry Hines Boulevard, Dallas, TX 75390, USA.

${ }^{7}$ These authors contributed equally to the work.

${ }^{8}$ Corresponding author.

E-MAIL scott@cmgm.stanford.edu; FAX (650) 725-7739.

Article and publication are at www.genesdev.org/cgi/doi/10.1101/ gad.869201. aries, the first evidence of the metameric organization of the embryo, between wg- and en/hh-expressing cells. At later stages of embryonic development, a tight balance between Wg and other signaling pathways, such as the Drosophila epidermal growth factor receptor (EGFR), determines whether epidermal cells secrete either naked (smooth) cuticle or hair-like structures called denticles (Dougan and DiNardo 1992; O'Keefe et al. 1997; Szuts et al. 1997). In the absence of $w g$ function, embryos are covered with a lawn of denticles, whereas otherwise wild-type embryos exposed to excess $\mathrm{Wg}$ produce a naked cuticle (Martinez Arias et al. 1988; Noordermeer et al. 1992).

Genetic and biochemical studies have lead to the identification of the key components of the Wnt/Wg pathway and have uncovered some of the molecular events that are involved in signal transduction (Fig. 1A). Wg binds 7-pass transmembrane receptors of the frizzled family (Fz or Dfz2), which, in turn, activate the cytoplasmic protein Dishevelled (Dsh; Klingensmith et al. 1994; Theisen et al. 1994; Bhanot et al. 1996). Dsh antagonizes the activity of a large protein complex that, in the absence of Wg signal, results in Armadillo (Arm)/ $\beta$-catenin phosphorylation and subsequent degradation by the ubiquitin-proteasome pathway (Yost et al. 1996; Aberle et al. 1997; Pai et al. 1997). This multiprotein complex includes Zw3/Glycogen synthase kinase $3 \beta$ (Gsk3 $\beta$ ), Adenomatous Polyposis Coli (APC), Axin, and Arm $/ \beta$ catenin. Axin constitutes the core of this complex, al- 

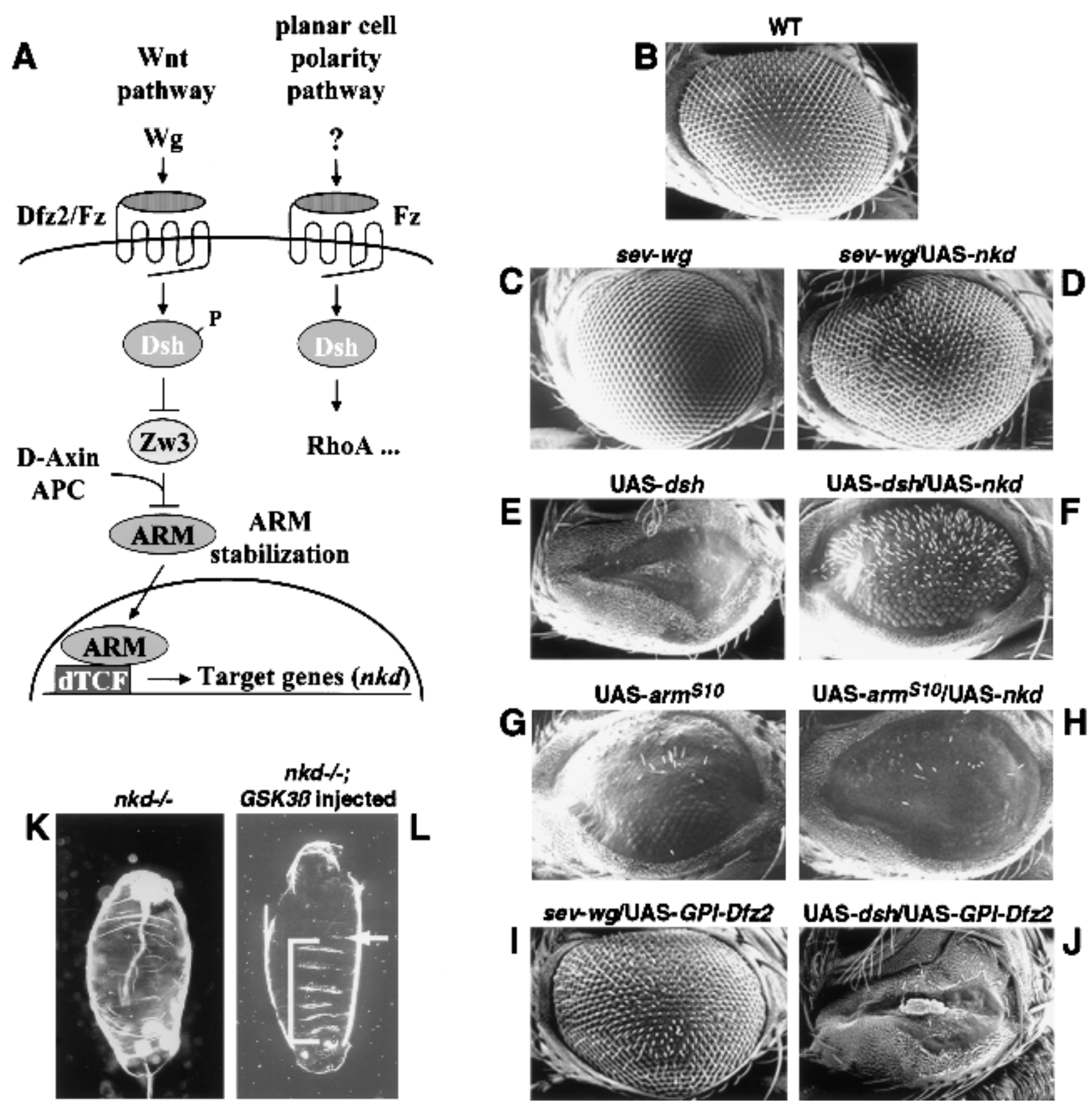

Figure 1. Epistasis study in the eye and embryo. (A) Schematic diagram of the Wg pathway and the planar cell polarity pathway. Arrows and bars show positive and negative actions, respectively. P represents the phosphorylated state of Dsh. See text for details. $(B-J)$ Nkd can suppress Wg and Dsh misexpression eye phenotypes, but not the $A_{r m}{ }^{S 10}$ misexpression eye phenotype. Ventral is to the left and anterior up. (B) The wild-type adult eye consists of an array of ommatidia and interommatidial bristles. $(C, E)$ The $s e v-W g$ or UAS- $d s h$ eyes lack bristles and/or ommatidia. $(G)$ The UAS- arm $^{S 10}$ eye has disrupted ommatidia and loss of bristles (average number of bristles/eye $=11.6 ; n=8) .(D, F)$ Co-misexpression of UAS- $n k d$ dramatically suppressed the $\operatorname{sev}-w g(n=8)$ and the UAS- $d s h(n=8)$ eye phenotypes. (H) Co-misexpression of UAS-nkd did not suppress the UAS- rrm $^{\text {s10 }}$ phenotype (average number of bristles/eye $=5.4$; $n=8)$. UAS-GPI-Dfz2 suppresses the sev-wg loss of bristle phenotype $(I)$ but does not suppress the UAS- $d s h$ phenotype $(J)$. $(K, L)$ Injection of Xenopus GSK3 $\beta$ mRNA into $n k d$ mutant embryos can restore denticles to the $n k d$ embryos. Anterior is up. ( $K) n k d$ mutant embryos lack ventral denticle belts. (L) Injection of $G S K 3 \beta$ into $n k d^{7 H 16}$ embryos restored ventral denticles (brackets). $n k d^{7 H 16}$ mutant embryos were identified by a $U b x$ mutation resulting in the transformation of the first abdominal segment A1 to the third thoracic segment T3 (arrow).

lowing Zw3/Gsk3 $\beta$ to phosphorylate Arm/ $\beta$-catenin (Ikeda et al. 1998). In the presence of $\mathrm{Wg}$ signal, Zw3 activity is reduced, resulting in stabilized Arm protein, which associates with the $\mathrm{dTCF} /$ Pangolin transcription 
factor to activate context-specific Wg target genes (Brunner et al. 1997; van de Wetering et al. 1997). In addition to transducing Wg signal, some of these components are involved in an Arm-independent pathway that regulates planar cell polarity (Fig. 1A; for review, see Shulman et al. 1998). During morphogenesis, this pathway orients cells in an axis orthogonal to their apical-basal axis by influencing the cytoskeleton (Shulman et al. 1998). It is required for the proper orientation of hairs and bristles on the thorax, abdomen, wing, and leg, as well as for the correct polarity of ommatidia in the eye. Both $\mathrm{Fz}$ and Dsh are involved in this pathway, but components downstream from Dsh are distinct from the Wg pathway (Axelrod et al. 1998; Boutros et al. 1998).

Given the Wnt/Wg pathway's key roles in cell growth and differentiation, it is not surprising to find that perturbations of its activity can lead to tumorigenesis. Integration of the mouse mammary tumor virus into the wnt-1 proto-oncogene locus promotes tumor formation in mice (Nusse and Varmus 1982). In addition, mutations in APC, $\beta$-catenin, and Axin that lead to aberrant Wnt activity have been associated with various types of human cancers (for review, see Polakis 2000). Inappropriate activation of Wnt target genes such as $c$-myc or cyclin D1 may be an important step toward tumor formation (Polakis 2000). It is therefore important to understand how Wnt signals are normally limited during development and cancer progression.

We recently reported the cloning of the naked cuticle $(n k d)$ gene and showed that $n k d$ antagonizes Wg signaling in Drosophila (Zeng et al. 2000). nkd Loss of function leads to embryonic lethality due to segmentation defects (Jürgens et al. 1984). The $n k d$ phenotype is characterized by a loss of ventral denticle belts and resembles the phenotype of embryos with excess Wg signaling, such as embryos overexpressing Wg or those lacking the negative regulators zw3, d-axin, or dAPC2 (Perrimon and Smouse 1989; Noordermeer et al. 1992; Hamada et al. 1999; McCartney et al. 1999). We also showed that the $n k d$ gene itself is regulated by $\mathrm{Wg}$, creating a negativefeedback loop that restricts Wg activity during segmentation (Zeng et al. 2000). Nkd is a novel protein containing a 60-amino acid region that is related to the high affinity $\mathrm{Ca}^{2+}$-binding EF-hand of the recoverin family of myristoyl switch proteins. Here we address how Nkd limits Wg signaling by using a combination of genetic and biochemical approaches. Our results suggest that Nkd can antagonize Wg signaling cell-autonomously through a direct interaction with Dsh.

\section{Results}

nkd regulates interommatidial bristle formation

The Drosophila eye is composed of mechanosensory bristles present at vertices of ommatidia (Fig. 1B). Bristle formation is suppressed near the circumferential margin of the eye, and the degree of suppression is least at the extreme dorsum of the head, typically 0-2 ommatidial diameters (Fig. 2A). Previous work showed that Wg sig- naling, active at the circumference of the developing eye where $w g$ is expressed, is responsible for this suppression of peripheral bristle formation (Cadigan and Nusse 1996; K.M. Cadigan et al., in prep.). To assay the function of $n k d$ in eye bristle formation, we used the EGUF/hid method (Stowers and Schwarz 1999) to make homozygous mutant $n k d$ eyes in $n k d /+$ animals. In this technique, Flp-mediated recombination between a chromosome mutant for $n k d$ and a chromosome harboring both recessive and dominant cell-lethal mutations is specifically induced in the eye using the eyeless promoter. During eye development, the only cells surviving are those that have lost the cell-lethal chromosome through recombination, producing an eye homozygous mutant for $n k d$. Examination of eyes mutant for the strong allele $n k d^{7 E 89}$ reveals, at the dorsum of the eye, consistent eye bristle suppression 3-5 ommatidial diameters away from the margin, with occasional closer bristles (Fig. 2B). This result suggests that endogenous $n k d$ regulates interommatidial bristle suppression by antagonizing the effects of endogenous Wg in cells farther than one cell diameter away from the Wg source.

\section{Nkd misexpression in the eye blocks Wg activity}

To determine how Nkd impinges on the Wg pathway, we tested the ability of Nkd to block the action of the positive regulators $\mathrm{Wg}$, Dsh, and Arm. To do so, we took advantage of a Drosophila eye misexpression system (Cadigan and Nusse 1996). Production of Wg in a subset of photoreceptor cells throughout the eye using a sevenless promoter transgene $(\mathrm{P}[\mathrm{sev}-\mathrm{wg}])$ prevents formation of interommatidial bristles in a paracrine fashion; otherwise, the eye is normal (Fig. 1C; Cadigan and Nusse 1996). Previous Nkd misexpression experiments did not indicate whether Nkd blocks Wg synthesis, Wg distribution, or cellular responses to received Wg (Zeng et al. 2000). To distinguish between these possibilities, we used the GAL4/UAS binary expression system to evaluate the effect of Nkd (UAS-nkd) on Wg-mediated eye bristle suppression. Misexpression of Nkd alone using multiple repeats of the eye-specific glass $(g l)$ enhancer (GMR) to drive the yeast transcription factor GAL4 (P[GMR-GAL4]) has no visible effect on eye development (data not shown). However, the combination of sev-wg with $n k d$ misexpression results in nearly complete suppression of the $\mathrm{P}[\mathrm{sev}$-Wg]-induced bristle-loss phenotype (Fig. 1D). Nkd misexpression did not alter the levels or distribution of $\mathrm{Wg}$ antigen (data not shown), indicating that Nkd is probably blocking signaling events downstream from Wg.

\section{Nkd misexpression blocks Dsh activity but not Arm activity}

The effect of Nkd on the downstream Wg pathway components Dsh and Arm was also tested using the GMRGAL4 system. Dsh misexpression (UAS-dsh) produces small, bristle-less eyes devoid of ommatidia (Fig. 1E). 

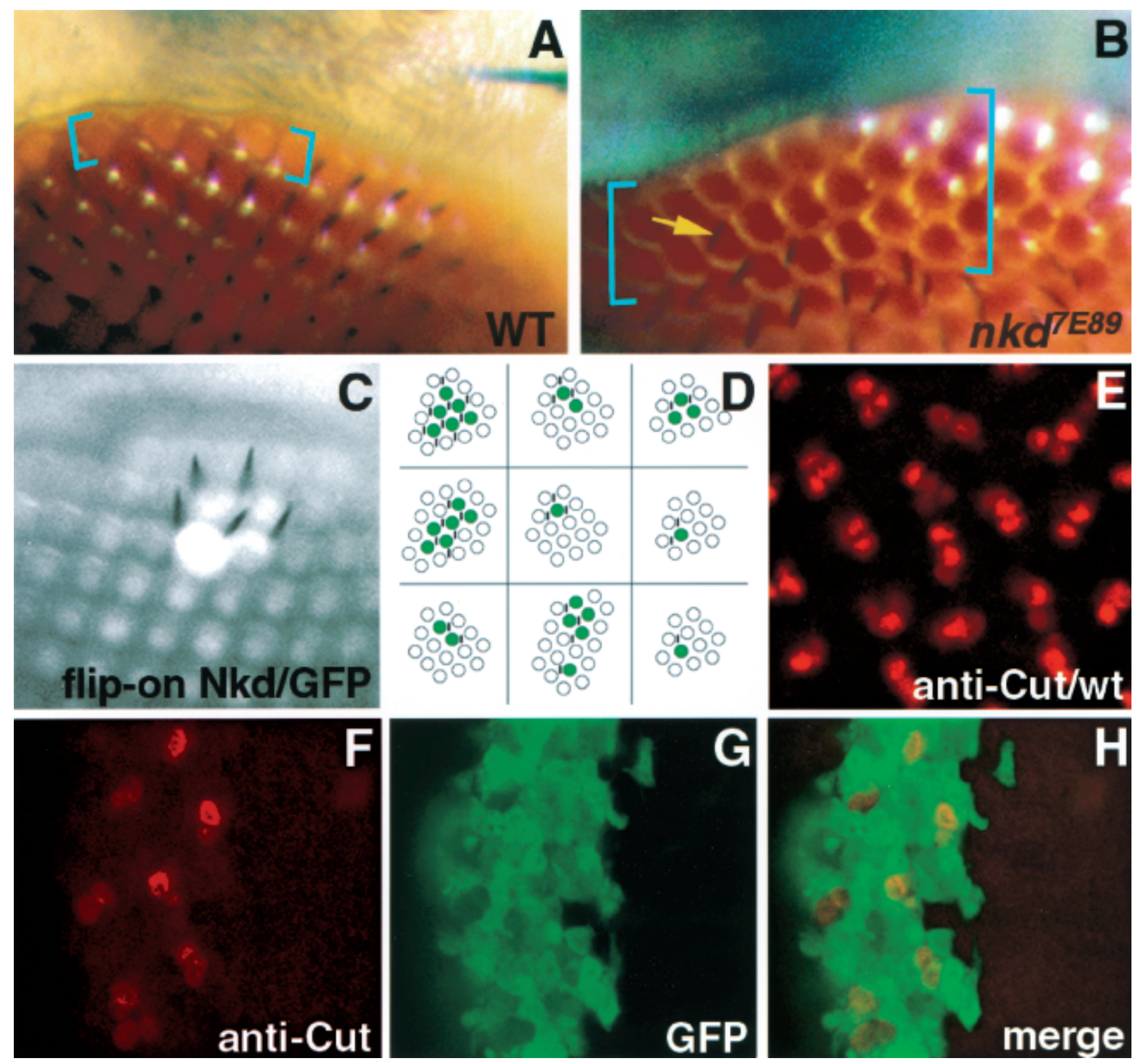

Figure 2. $n k d$ autonomously regulates interommatidial bristle formation. (A) Wild-type eye margin at dorsum of head shows bristle suppression 0-1 ommatidial rows (blue brackets) from eye margin. (B) nkd ${ }^{7 E 89}$ mutant eye shows suppression of bristles 3-5 ommatidial rows from eye margin. Occasional bristles are present closer to eye margin (yellow arrow). No bristle phenotype was seen with the weaker $n k d^{9 G 33}$ allele, whereas $h^{1} n k d^{7 H 16}$ eyes are small and rough (not shown), possibly due to $h / n k d$ interactions (Zeng et al. $2000)$ and hence could not be scored for this phenotype. $(C, D)$ Nkd/GFP misexpression clones in sev-wg adult eyes. $(C)$ Bristles are restored only in the region of the clone marked by GFP. (D) Cartoon depiction of nine adult eye clones (green color); vertical black lines represent bristles. $(E)$ Cut nuclear localization in bristle cells of a wild-type pupal eye disc. $(F, G)$ Restoration of bristle cells in a sev-Wg pupal eye, revealed by Cut localization (red color), is confined to the region of the Nkd/GFP misexpression clone (green color); the clone begins at the edge of the disc (left) and continues inward (right). $(H)$ Merged image of $F$ and $G$ shows that GFP and Cut colocalize in bristle cell precursor nuclei present in the clone.

Nkd strongly suppressed the Dsh misexpression eye phenotype, restoring numerous bristles and ommatidia (Fig. $1 \mathrm{~F})$. If the Dsh misexpression eye phenotype is Wg-dependent, its suppression by Nkd could be due to Nkd acting on Wg rather than on Dsh or other downstream components. Previous work suggests that the Dsh mis- expression eye phenotype is Wg-independent (Cadigan and Nusse 1996). To confirm the Wg-independence of the Dsh phenotype, a dominant-negative form of Dfz2 (UAS-GPI-Dfz2; Cadigan et al. 1998) was coexpressed with either sev-wg or UAS-dsh. UAS-GPI-Dfz2 effectively suppressed sev-Wg-induced bristle loss in the eye 
(Fig. 1I). Coexpression of UAS-GPI-Dfz2 and UAS-Dsh resulted in some eye necrosis, but it had negligible effects on the UAS- $d s h$ eye phenotype (Fig. 1J). These results confirm that the Dsh misexpression effect in the eye is Wg-independent. Therefore, rescue of the UAS- $d s h$ phenotype by Nkd is not an indirect effect due to suppression of Wg activity.

GMR-driven expression of UAS- $\mathrm{arm}^{\mathrm{S1O}}$, a constitutively activated form of arm, also produces bristle loss and failure of proper ommatidial development (Fig. 1G; Ahmed et al. 1998). Nkd coexpression had no effect on the Arm misexpression phenotype (Fig. 1H). Dsh and Arm misexpression phenotypes were not affected by simultaneous expression of UAS-lacZ, indicating that suppression of the dominant eye phenotypes by Nkd was not due to GAL4 titration (data not shown). The ability of Nkd to block effects of Wg and Dsh but not Arm suggests that Nkd is acting at the level of, or downstream from, Dsh but not downstream of Arm.

\section{Epistasis between nkd and zw3 in the embryo}

The relationship between Nkd and Zw3 could not be determined by a similar suppression test because both proteins are negative regulators of $\mathrm{Wg}$. In addition, the subtlety of the $n k d$ phenotype in the eye made this tissue unsuitable for analyzing the epistasis between $n k d$ and $z w 3$. Instead, Zw3/Gsk3 $\beta$ was overproduced in $n k d$ mutant embryos using genetic and mRNA injection methods: We used heat shock promoter (hsp70)-controlled GAL4 to drive Zw3 production, or we used injections with Xenopus gsk3 $\beta$ mRNA. Vertebrate gsk3 $\beta$ genes have sequences very similar to the fly gene and can rescue zw3 mutant embryos (Siegfried et al. 1992). nkd mutants lack ventral denticle belts and are considerably smaller than wild-type embryos (Fig. 1K). Overproduction of Gsk3 $\beta$ or Zw3 in $n k d$ mutants results in partial to almost complete restoration of denticle belts and restoration of more normal embryo size (Fig. 1L; data not shown). Because Zw3 restores denticles to nkd mutants, Zw3 cannot act genetically upstream of the defect in $n k d$ mutants (i.e., by stimulating $n k d$ function) in the linear Wg pathway. Nkd therefore is likely to act upstream of, or in a pathway parallel to, Zw3 and downstream from, or at the level of, Dsh.

\section{Nkd acts cell-autonomously}

The eye misexpression results suggest that Nkd antagonizes Wg signaling at the level of, or downstream from, Dsh. Loss-of-function $d s h$ clones revealed that Dsh acts autonomously in Wg-responsive cells (Klingensmith et al. 1994), suggesting that Nkd must also act in Wg-responsive cells. Indeed, previous observations in fly embryos suggest an initial requirement for $n k d$ in cells receiving the Wg signal (Martinez Arias et al. 1988; Dougan and DiNardo 1992; Zeng et al. 2000). Because eye development allows fairly easy production of sharply bounded clones, we chose the eye to assess the cell au- tonomy of Nkd action. Marked clones of Nkd-misexpressing cells were produced in developing eyes and the range of Nkd action on $s e v-W g$ was monitored.

We used the flip-on GAL4 system to make random clones of cells misexpressing both Nkd and a cell-autonomous marker, green fluorescent protein (GFP), in eyes with excess $\mathrm{Wg}$ (sev-Wg eyes). All clones examined $(n=15)$ showed suppression of bristle loss, with the suppression consistently within or immediately adjacent to GFP misexpression clones (Fig. 2C,D). No bristles were present outside the clones, indicating a local action of Nkd. To address whether Nkd was acting only in bristle precursor cells, and hence cell-autonomously, we specifically marked those cells with antisera against the Cut nuclear protein in pupal eye discs /Cadigan and Nusse 1996). In the vicinity of Nkd misexpression clones, there was a perfect correlation between GFP and Cut-labeled cells: All Cut-positive bristle precursor cells expressed GFP and hence Nkd; no GFP-negative/Cut-positive cells were found (Fig. 2F-H; $n=8$ clones). These results suggest that Nkd was acting within Cut-positive bristle precursor cells to antagonize the inhibitory effects of Wg on bristle cell differentiation.

The dsh; nkd double-mutant resembles dsh mutant embryos

Cuticles derived from embryos lacking wg activity ( $w g$, $d s h$, or arm) have nearly continuous fields of denticles, whereas HS-wg embryos, or those mutant for the negative regulator $z w 3$, secrete naked cuticle (Martinez Arias et al. 1988; Perrimon and Smouse 1989; Noordermeer et al. 1992). Wg misexpression and double-mutant analyses showed that Wg acts sequentially through Dsh, Zw3, and Arm (Siegfried et al. 1992, 1994; Noordermeer et al. 1994; Peifer et al. 1994). Embryos doubly mutant for wg and $z w 3(z w 3 ; w g)$, as well as zw3 dsh embryos, resemble zw3 embryos, whereas zw3 arm embryos resemble arm embryos, indicating that zw3 acts downstream from $d s h$ and upstream of arm (Siegfried et al. 1992, 1994; Peifer et al. 1994). Mutations in either $n k d$ or $z w 3$ give rise to a naked cuticle phenotype, with posterior expansion of en expression and ectopic $w g$ expression in the developing embryo (Martinez Arias et al. 1988; Perrimon and Smouse 1989). However, in contrast to the naked cuticle phenotype of the $z w 3$; wg embryo, the $w g$; $n k d$ embryo has a wg-like phenotype (Bejsovec and Wieschaus 1993), indicating a dependence on Wg for the naked cuticle phenotype of $n k d$ mutants (Dougan and DiNardo 1992).

To clarify the relationship between Nkd and other Wg pathway components in the embryo, we made embryos doubly mutant for $n k d$ and $d s h$ or arm, using both genetic means and RNA interference (RNAi). Whereas the $n k d$ gene is strictly zygotic (Zeng et al. 2000), dsh has a maternal contribution that must be removed via germline clones to obtain the embryonic $d s h$ phenotype (Perrimon and Mahowald 1987). Females heterozygous for $n k d$ and carrying $d s h$ germ-line clones were crossed to males heterozygous for $n k d$ (see Materials and Methods). 
Embryos derived from crosses using different combinations of $n k d$ and $d s h$ alleles were counted and grouped according to their cuticle phenotypes (Table 1). The expected Mendelian ratios are $37.5 \%$ wild type (3/8), 37.5\% dsh $(3 / 8), 12.5 \%$ nkd $(1 / 8)$, and $12.5 \%$ dsh; nkd $(1 / 8)$. Only three phenotypes could be detected-wild type, $d s h$, and $n k d$-indicating that the $d s h ; n k d$ mutants exhibit one of these phenotypes or die before secreting cuticle. Whereas the observed percentages for the wild-type and $n k d$ categories are very close to the expected percentages $(38.2 \%$ and $14.3 \%$, respectively), the percentage of $d s h$ embryos is significantly higher $(47.5 \%)$, suggesting that the $d s h ; n k d$ mutant resembles the $d s h$ mutant.

To confirm the $d s h$; nkd phenotype, we performed RNAi experiments. Injection of nkd double-stranded RNA (dsRNA) into wild-type embryos efficiently mimics $n k d$ loss of function: $76 \%$ of the injected embryos $(n=221)$ develop with greatly reduced denticles compared to wild-type embryos (Fig. 3A,B). The majority of these mutant embryos $(69 \%)$ show an intermediate to strong $n k d$ cuticle phenotype, the others showing a weak expressivity characterized by a loss of only a few denticles (data not shown). We also tried RNAi with $d s h$, but, in contrast to $n k d$, both the penetrance and the expressivity of the $d s h$ phenotype were very weak (data not shown). Increasing the $d s h$ dsRNA concentration had little effect, producing only a fusion between belts A4 and A5 in $<5 \%$ of the injected embryos and ruling out the utility of $n k d$ and $d s h$ double injections. Instead we injected $n k d$ dsRNA into $d s h$ embryos derived from germline clones. Half of the collected embryos are wild type due to rescue by the paternal X-chromosome (see Materials and Methods for the cross). To score only nonrescued $d s h$ mutant embryos, we crossed females carrying germ-line clones to males carrying an X-chromosome GFP balancer and scored GFP-negative embryos after eliminating GFP embryos. Injection of $n k d$ dsRNA into $d s h$ embryos had no effect on the $d s h$ phenotype $(n=66$; data not shown), confirming that $d s h$; nkd double mutants resemble $d s h$ embryos.

\section{arm; nkd double-mutant embryos resemble arm} embryos

We used the null allele arm $^{\text {YD35 }}$ (Peifer and Wieschaus 1990) to generate arm; nkd double-mutant embryos. Em- bryos homozygous for this allele have a strong arm phenotype, even without making germ-line clones. Male heterozygotes for the strong alleles $n k d^{7 H 16}$ or $n k d^{7 E 89}$ were crossed to females heterozygous for $\operatorname{arm}^{Y D 35}$ and $n k d^{7 H 16}$ (see Materials and Methods). As these crosses generate a majority of wild-type embryos (a ratio of nine wild type to seven mutants), we counted only cuticles from unhatched embryos, which are expected to be mutant for arm (ratio 3:7, 42.9\%), nkd (3:7, 42.9\%), and arm; $n k d(1: 7,14.3 \%)$. Like the $d s h ; n k d$ embryos, the arm; $n k d$ mutants do not exhibit a distinct phenotype. The results (Table 2) show that the $n k d$ phenotype is found at the expected frequency $(42.6 \%)$, but the arm phenotype is over-represented $(57.4 \%$ instead of $42.9 \%)$, indicating that this category also contains the arm; nkd embryos. Therefore, the arm; nkd mutant is covered with denticles and resembles arm embryos.

The double-mutant analysis indicates that the nkd phenotype occurs only if $w g$, $d s h$, and arm genes are active, confirming the requirement for $\mathrm{Wg}$ signaling to generate the $n k d$ phenotype (Dougan and DiNardo 1992; Bejsovec and Wieschaus 1993). Zw3 constitutively represses $\mathrm{Wg}$ target-gene transcription, and the role of $\mathrm{Wg}$ is to overcome this inhibition (Siegfried et al. 1992). Our results indicate that Nkd, in contrast, is required to oppose Wg signal. Removal of $n k d$ in the absence of $w g$, $d s h$, or arm has little effect on cuticle phenotype. Accordingly, increased levels of Nkd do not modify the $w g$ mutant cuticle (Zeng et al. 2000). The negative influence of Nkd could be mediated by inhibition of Dsh activity, stimulation of Zw3 activity, or by interactions with unknown pathway components. To test whether Nkd can directly interact with known Wg signaling components, we used yeast two-hybrid and in vitro binding assays.

\section{Nkd directly interacts with Dsh in yeast and in vitro}

Expression in yeast of full-length Nkd protein fused to the GAL4 DNA-binding domain (GB-Nkd) did not activate transcription by itself (Fig. 4A). When GB-Nkd was coexpressed with Dsh fused to the activation domain of GAL4 (GAD-Dsh), strong $\beta$-galactosidase activity was detected, indicating an interaction between Nkd and Dsh (Fig. 4A). The reverse experiment, using GB-Dsh and GAD-Nkd, could not be performed because GB-Dsh ac-

Table 1. The dsh; nkd double-mutant resembles dsh embryo

\begin{tabular}{llllr}
\hline$q \times \delta$ & \multicolumn{1}{c}{ wt } & \multicolumn{1}{c}{$d s h$} & nkd & No. of embryos \\
\hline$d s h^{v 26} ; n k d^{7 H 16} \times n k d^{7 H 16}$ & $38.7 \%(144 / 140)$ & $47.6 \%(177 / 140)$ & $13.7 \%(51 / 146)$ & 372 \\
$d s h^{v 26} ; ; n k d^{7 H 16} \times n k d^{7 E 89}$ & $40.2 \%(181 / 169)$ & $45.6 \%(205 / 169)$ & $14.2 \%(64 / 56)$ & 450 \\
$d s h^{v 26} ; n k d^{7 E 89} \times n k d^{7 H 16}$ & $32.2 \%(75 / 87)$ & $50.2 \%(117 / 87)$ & $17.6 \%(41 / 29)$ & 233 \\
$d s h^{477} ; n k d^{7 H 16} \times n k d^{7 H 16}$ & $39.2 \%(98 / 94)$ & $48.4 \%(121 / 94)$ & $12.4 \%(31 / 31)$ & 250 \\
Total & $\mathbf{3 8 . 2 \% ( 4 9 8 / 4 8 9 )}$ & $\mathbf{4 7 . 5 \% ( 6 2 0 / 4 8 9 )}$ & $\mathbf{1 4 . 3 \% ( 1 8 7 / 1 6 3 )}$ & 1305 \\
\hline
\end{tabular}

Phenotypic distribution of embryos laid by $d s h /$ ovo $^{D 1} ;$;nkd/+ females ( $q$ ) mated to $+/ Y ; n k d / T M 3$ males ( $\hat{0}$; see Materials and Methods for details). Four cuticular phenotypes were expected: wild-type (wt), $d s h$, nkd and unknown corresponding to the $d s h$; $n k d$ double mutant, with ratios of $3: 8(37.5 \%), 3: 8(37.5 \%), 1: 8(12.5 \%)$ and $1: 8(12.5 \%)$, respectively. Two $d s h$ alleles $\left(d s h^{\text {v26 }}, d s h^{477}\right)$ and two $n k d$ alleles $\left(n k d^{7 H 16}, n k d^{7 E 89}\right)$, all strong alleles, were used in different combinations, as indicated in the first column. Results are presented as percentages followed between parentheses by the observed number of embryos (first number) and the expected number (second number). A total of 1305 embryos were counted and the total percentage for each phenotype is indicated in bold. 

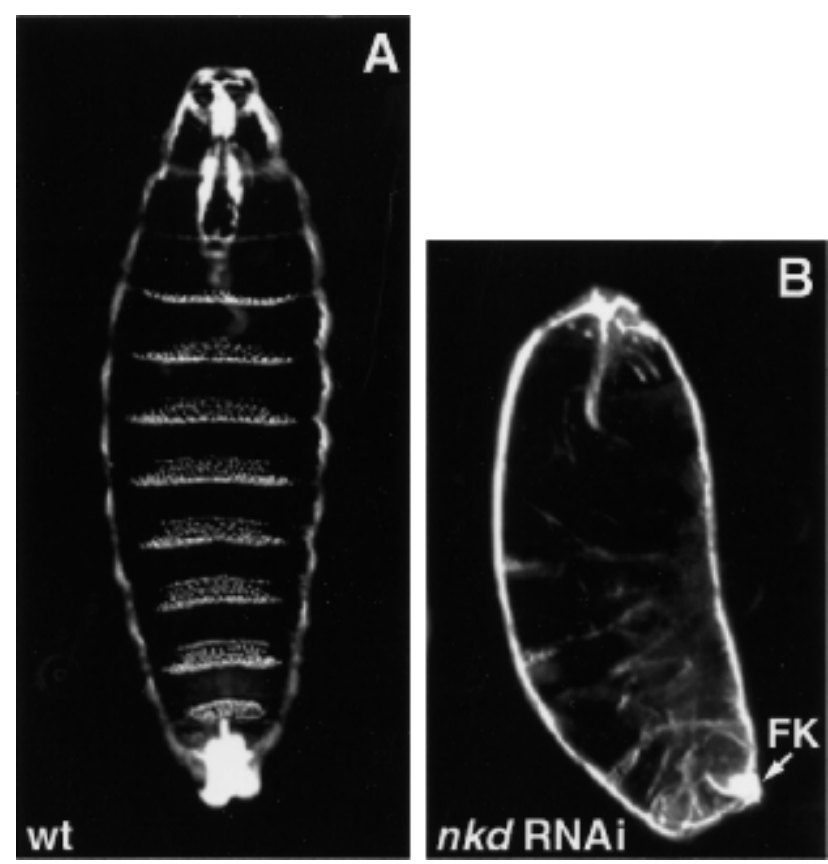

Figure 3. Cuticle phenotypes of embryos injected with $n k d$ dsRNA. (A) Wild-type (wt) embryo shortly before hatching. The ventral side is characterized by denticle belts separated with naked cuticle. (B) nkd RNAi mimics the $n k d$ phenotype. Embryos are shorter and lack denticle belts, resembling $n k d^{-1-}$ embryos (see Fig. 1K). nkd RNAi embryos also show the characteristic defects in the Filzkörper (FK).

tivates transcription on its own (data not shown). No interaction between Nkd and Zw3 was detected, nor did Nkd interact with the C-terminal intracellular portion of Dfz2 (Dfz2CT), Arm, or the control protein large T-antigen (T-Ag; Fig. 4A). Nkd also did not interact with DAxin (data not shown).

The interaction between Dsh and Nkd was confirmed using coimmunoprecipitation and glutathione-S-transferase (GST) pull-down assays. For coimmunoprecipitation tests, COS-7 cells were transfected with vectors expressing Myc-tagged Nkd and Dsh proteins. Immunoprecipitation with anti-Dsh, followed by protein blotting with anti-c-Myc antibody, showed that Nkd and Dsh can be coprecipitated (Fig. 4B). For GST pull-down assays, in vitro translated $\left[{ }^{35} \mathrm{~S}\right]$ methionine Dsh bound to GST-Nkd fusion protein but not to GST or Luciferase protein (Fig. 4C). Similarly, in vitro translated $\left[{ }^{35} \mathrm{~S}\right]$ methionine-la- beled Nkd specifically bound GST-Dsh (Fig. 4D). All three protein association assays indicate that $\mathrm{Nkd}$ and Dsh can directly interact, in keeping with the epistasis results that suggested a role for Nkd at the level of Dsh or Zw3.

Production of Nkd-GFP fusion protein in larval salivary glands revealed striking colocalization with endogenous Dsh, stained with an anti-Dsh antibody (data not shown), indicating that the two proteins may also interact in vivo. However, we did not detect an association between the two proteins using coimmunoprecipitation experiments with embryo extracts or lysates from Drosophila cell lines (data not shown). The negative results may be due to protein complex dynamics, accessibility to antibodies, low levels of the complex in fly cells, as well as possible modes of regulation of the interaction, which we are currently investigating.

\section{Nkd interacts with the basic/PDZ region of Dsh}

The Dsh protein contains three defined domains: DIX, PDZ, and DEP (Fig. 5D). The DIX (Dishevelled, Axin) domain shares homology with the C-terminal part of DAxin, the PDZ (PSD-95, Dlg, Zo-1) domain is a modular region involved in protein-protein interactions, and the DEP (Dsh, Egl-10, Pleckstrin) domain is usually found in signaling proteins, although its role remains unclear. In addition, a stretch of basic residues is present between the DIX and PDZ domains. Using both the yeast twohybrid system and GST pull-down experiments, the region in Dsh that binds Nkd was defined (Fig. 5). Our results indicated that the central region of Dsh containing the basic sequence and the PDZ domain was sufficient for binding Nkd (Fig. 5D). The PDZ domain of Dsh is necessary for the interaction with Nkd but, in contrast to proteins such as casein kinase I or Frat1 (Li et al. 1999; Peters et al. 1999), it cannot efficiently bind Nkd by itself (Fig. 5D).

\section{Nkd overexpression specifically affects Dsh function in planar cell polarity}

Dsh is a branchpoint connecting two distinct signaling pathways in Drosophila development: the Wg pathway and the planar cell polarity pathway (PCP; Boutros and Mlodzik 1999|. nkd mutant clones have normal planar cell polarity (Zeng et al. 2000); so there is no detectable

Table 2. The arm; nkd double-mutant resembles arm embryos

\begin{tabular}{|c|c|c|c|}
\hline$q \times 0$ & $\mathrm{arm}$ & $n k d$ & $\begin{array}{c}\text { No. of } \\
\text { unhatched embryos }\end{array}$ \\
\hline $\operatorname{arm}^{\text {YD35 }} ; n k d^{7 H 16} \times n k d^{7 H 16}$ & $58.0 \%(677 / 500)$ & $42.0 \%(490 / 500)$ & 1167 \\
\hline $\operatorname{arm}^{Y D 35} ; n k d^{7 H 16} \times n k d^{7 E 89}$ & $56.6 \%(571 / 432)$ & $43.4 \%(437 / 432)$ & 1008 \\
\hline Total & $\mathbf{5 7 . 4 \%}(1248 / 932)$ & $\mathbf{4 2 . 6 \%}(927 / 932)$ & 2175 \\
\hline
\end{tabular}

Phenotypic distribution of unhatched embryos laid by $\operatorname{arm}^{Y D 35} /+; n k d^{7 H 16} /+$ females $(q)$ crossed with $+/ Y_{; ; n k d^{7 H 16} / T M 3}$ or $+/ Y_{; ; n k} d^{7 E 89} /$ TM3 males ( $\delta$ ). Three phenotypes were expected among the unhatched embryos: arm, $n k d$ and $a r m ; n k d$, the latter being unknown. The predicted ratios are: $3: 7(42.9 \%)$ for arm and $n k d$, and 1:7 (14.3\%) for arm; nkd. Results are presented as in Table 1 . 

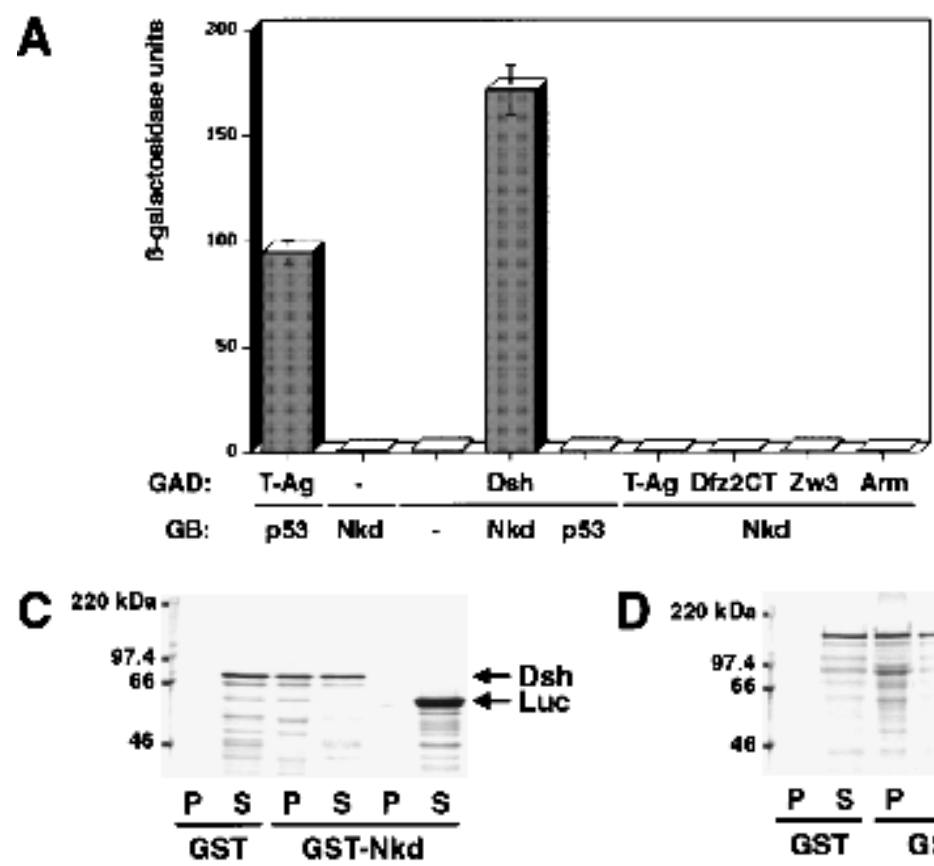

D $220 \mathrm{kDn}$.

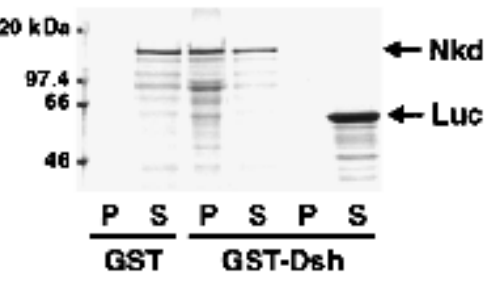

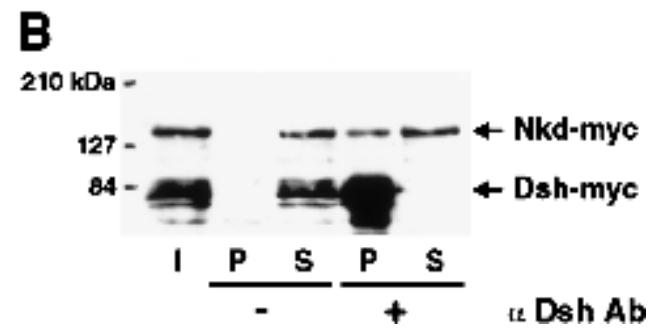

Figure 4. Nkd and Dsh directly interact in the yeast two-hybrid system, coimmunoprecipitation and GST pulldown assays. (A) Interaction between Nkd and Dsh in the yeast two-hybrid system using a liquid culture $\beta$-galactosidase assay. Yeast cells were cotransformed with plasmids expressing the GAL4 DNA-binding domain (GB, amino acids [aa] 1-147) either alone (-) or in fusion with Nkd, along with plasmids expressing the GAL4 activation domain (GAD, aa 768-881) alone (-) or in fusion with Dsh. The interaction between GB-Nkd and GAD-Zw3, GAD-

Arm, or GAD-Dfz2CT (intracellular portion of Dfz2) was also tested. Murine p53 (aa 72-390) fused to GB and SV40 large T-antigen (T-Ag, aa 87-708) fused to GAD were used as positive controls. For each transformation, results corresponding to the mean of 2-independent yeast colonies are shown. The values are expressed in Miller units. The activation was confirmed using a second reporter gene (ADE2) present in the yeast strain and protein blots of yeast extracts showed that the different fusion proteins accumulated to similar levels (data not shown). (B) Coimmunoprecipitation of Nkd and Dsh in COS-7 cells. Extracts from COS-7 cells expressing Nkd-myc and Dsh-myc were subjected to coimmunoprecipitation with anti-Dsh antibody $(\alpha \mathrm{Dsh} \mathrm{Ab})(+)$. As a control, the anti-Dsh antibody was omitted $(-)$. The eluted protein from the beads $(\mathrm{P})$ and one-tenth of the supernatant $(\mathrm{S})$, as well as proteins corresponding to one-tenth of the input (I) were analyzed by Western blot using anti-c-Myc antibody. $(C, D)$ Interaction between Nkd and Dsh in the GST pull-down assay. Bacterially expressed GST-Nkd protein was incubated with $\left[{ }^{35}\right.$ S]methionine-Dsh, produced and labeled by in vitro translation $(C)$, whereas GST-Dsh was incubated with $\left[{ }^{35}\right.$ S]methionine-Nkd $(D)$. As controls, GST-Nkd or GST-Dsh proteins were also tested for their interaction with $\left[{ }^{35} \mathrm{~S}\right]$ methionine-Luciferase (Luc). The eluted proteins from the beads $(\mathrm{P})$ and one-tenth of the supernatant (S) were separated by SDS-PAGE and viewed using a phosphorimager (Molecular Dynamics).

normal role for $n k d$ in the PCP pathway. If Nkd affects Dsh during Wg signaling, as our data suggest, then appropriately timed overexpression of Nkd might be able to specifically alter Dsh function in PCP signaling. Nkd was tested for its ability to interfere with PCP signaling during a time when $\mathrm{Fz}$ and Dsh are not appreciably participating in Wg signaling. Timed overexpression of Nkd at $24 \mathrm{~h}$ after puparium formation (APF) produces adult flies with wing hair polarity defects that are indistinguishable from those seen in $d s h^{1}$ mutant adults (Fig. $6 \mathrm{~A}-\mathrm{C}) . d s h^{1}$ is an adult viable allele of $d s h$ that harbors a missense mutation in the C-terminal DEP domain (Axelrod et al. 1998; Boutros et al. 1998). Genetic tests have shown $d s h^{1}$ to be a null allele for PCP signaling (Perrimon and Mahowald 1987). The Nkd overexpression polarity pattern is reproducible and qualitatively distinct from that produced by complete loss of function of other known PCP mutants, including $f z$ or prickle ( $p k$; Fig. 6D,E; Gubb and Garcia-Bellido 1982). The Nkd overexpression defect is also different from those associated with Fz or Dsh overexpression (data not shown).

The PCP phenotype associated with Fz overexpression is sensitive to the dose of $d s h$ (Krasnow and Adler 1994). To determine whether Nkd could similarly titrate Dsh from PCP signaling induced by $\mathrm{Fz}$ overexpression, we simultaneously expressed $\mathrm{Fz}$ and Nkd. Indeed, overexpressed Nkd suppressed the effects of excess Fz (Fig. 6FH). Neither excess Nkd nor decreased nkd dosage modified the wing bristle polarity of $d s h^{1}$ mutant flies (data not shown). The results suggest that Nkd can specifically interfere with Dsh function in planar cell polarity and that this effect requires wild-type Dsh protein.

\section{Discussion}

Segment polarity genes encode signaling proteins that establish cell fate within each segment of the Drosophila embryo. A positive feedback loop between the Wg and Hh signaling pathways, active in adjacent cells, specifies parasegmental boundaries during early segmentation. Previous work showed that $n k d$ is necessary to restrict the expression of the $\mathrm{Wg}$ target gene en as soon as its expression becomes dependent on $\mathrm{Wg}$ (Bejsovec and Wieschaus 1993; van den Heuvel et al. 1993). The inducible antagonist role of $\mathrm{Nkd}$ creates a negative feedback loop that limits Wg signaling during early embryogenesis (Zeng et al. 2000). Here we provide experimental evidence that $n k d$ also regulates $\mathrm{Wg}$ activity in the eye. Our 
Figure 5. Nkd interacts with the basic/PDZ region of Dsh. (A) Interaction of different Dsh deletion mutants, fused to GAD, with GB or GB-Nkd in the yeast two-hybrid system. Yeast growth was evaluated using the ADE reporter gene present in the strain: Yeast colonies expressing the different combinations of fusion proteins, as indicated, were grown on medium containing $(+)$ or lacking $(-)$ adenine. $(B, C)$ Using the GST pull-down assay, $\left[{ }^{35} \mathrm{~S}\right]$ methionine-Dsh D1 to D6 were tested for their capacity to bind GST-Nkd $(B)$, whereas in $(C)$, GST-DshD6PDZ and GST-DshPDZ proteins were incubated with $\left[{ }^{35} \mathrm{~S}\right]$ methionine-Nkd. The curved bands observed with D1 and D6 are due to comigration with a byproduct of GST-Nkd protein (data not shown). (D) Summary of domain mapping results from the yeast two-hybrid system (Y2H) and the GST pull-down assay (GST). (+) Positive interaction, $(-)$ no interaction, $(+/-)$ weak interaction, (n.d.) not determined. The different domains of Dsh, DIX, basic region (b), PDZ, and DEP, are indicated.
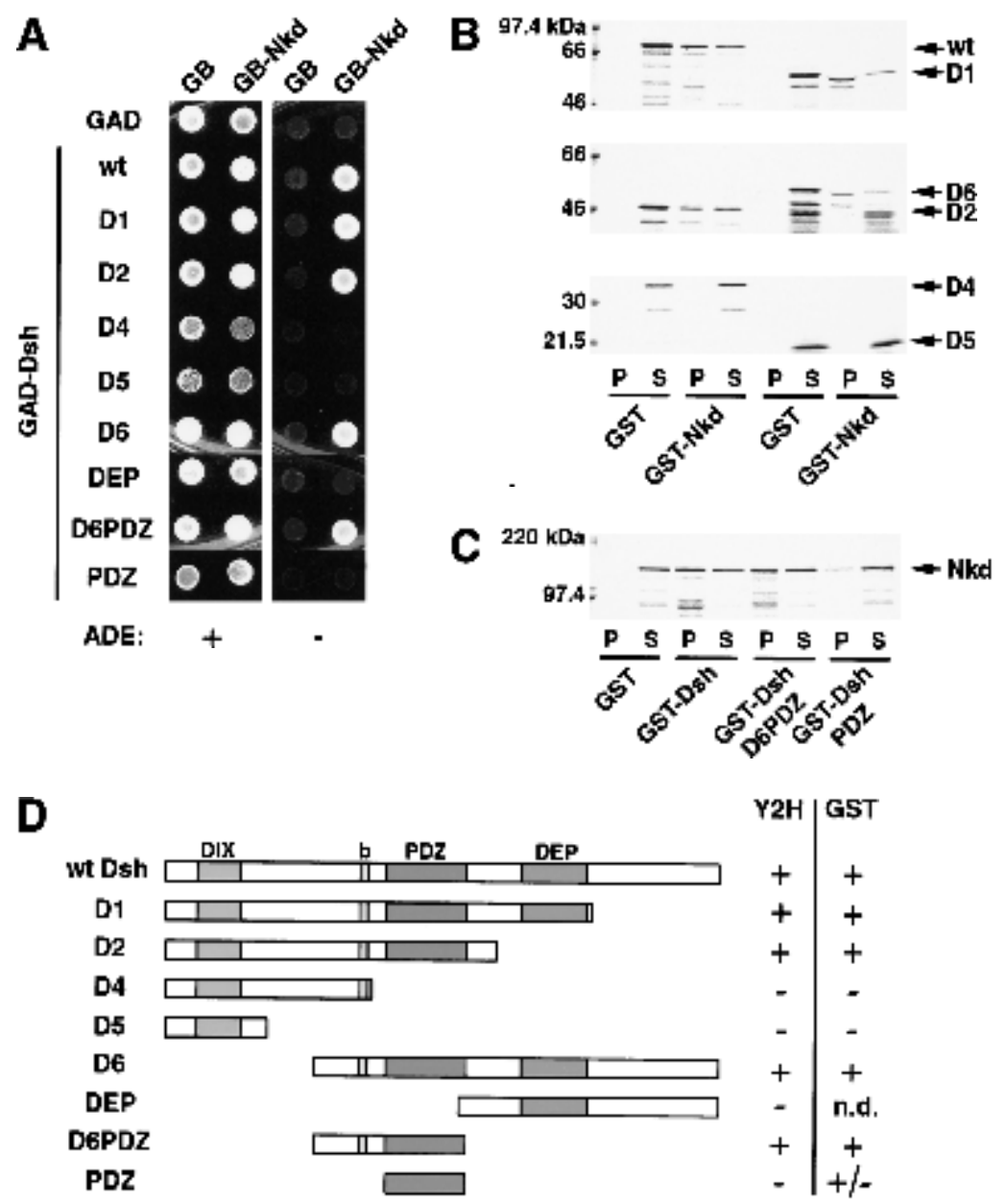

epistasis study suggests that Nkd acts cell-autonomously to antagonize $\mathrm{Wg}$ signaling at the level of, or between, Dsh and Zw3. In agreement with this study, yeast and biochemical experiments reveal that Nkd can bind directly to Dsh. Misexpressed Nkd specifically phenocopies loss of $d s h$ in planar cell polarity, showing that Nkd can specifically interfere with endogenous $d s h$ function.

Our data suggest a model of how Nkd limits early Wg signaling in the embryo. At stage 8-9 of wild-type embryonic development, when early segmental patterning events are taking place, $\mathrm{Wg}$ protein maintains the transcription of en/hh in adjacent posterior cells (Klingensmith and Nusse 1994). Cells located farther posterior receive an insufficient amount of $\mathrm{Wg}$ for induction of en/hh expression (Vincent and O'Farrell 1992). In nkd mutants at this stage, the more posterior cells ectopically express en/hh in a fashion that is Wg-dependent (Bejsovec and Wieschaus 1993; van den Heuvel et al. 1993). When ectopic en/hh transcription is first observed in $n k d$ mutants at stage $8-9, \mathrm{Wg}$ distribution is apparently normal (Moline et al. 1999; data not shown), indicating that Nkd normally prevents more distant cells from turning on en/hh in response to $\mathrm{Wg}$. Consistent with this requirement, we saw enhanced $n k d$ expression precisely in these more distant cells during stage 9 (Zeng et al. 2000). Our present results suggest that Nkd may act through Dsh in those cells to block Wg activity. Later in development, at stage 10-11, inappropriate Wg distribution may also contribute to the $n k d$ phenotype (Moline et al. 1999). By stage 11, an ectopic stripe of $w g$ expression is induced just posterior to the expanded en/ hh domain and is required for the excess naked cuticle seen in $n k d$ mutants (Dougan and DiNardo 1992). This extra $w g$ stripe depends on $\mathrm{Wg}$ and $\mathrm{Hh}$ activities, as well as on the action of the pair-rule transcription factors Sloppy paired (Bejsovec and Wieschaus 1993; van den Heuvel et al. 1993; Cadigan et al. 1994).

Our experiments further clarify the differences between Nkd and Zw3 in Wg signaling. Embryos lacking both maternal and zygotic $z$ W3 have a naked cuticle phenotype and expanded stripes of en transcription as in $n k d$ mutants (Perrimon and Smouse 1989; Siegfried et al. 1992). Both Zw3 and Nkd are negative regulators of en transcription (Martinez Arias et al. 1988; Siegfried et al. 1992), but en stripe expansion is independent of Wg signaling in zw3 mutants and Wg dependent in nkd mutants (Siegfried et al. 1992; Bejsovec and Wieschaus 1993; van den Heuvel et al. 1993). Cuticles derived from double-mutant embryos illustrate the difference: $z w 3$; 

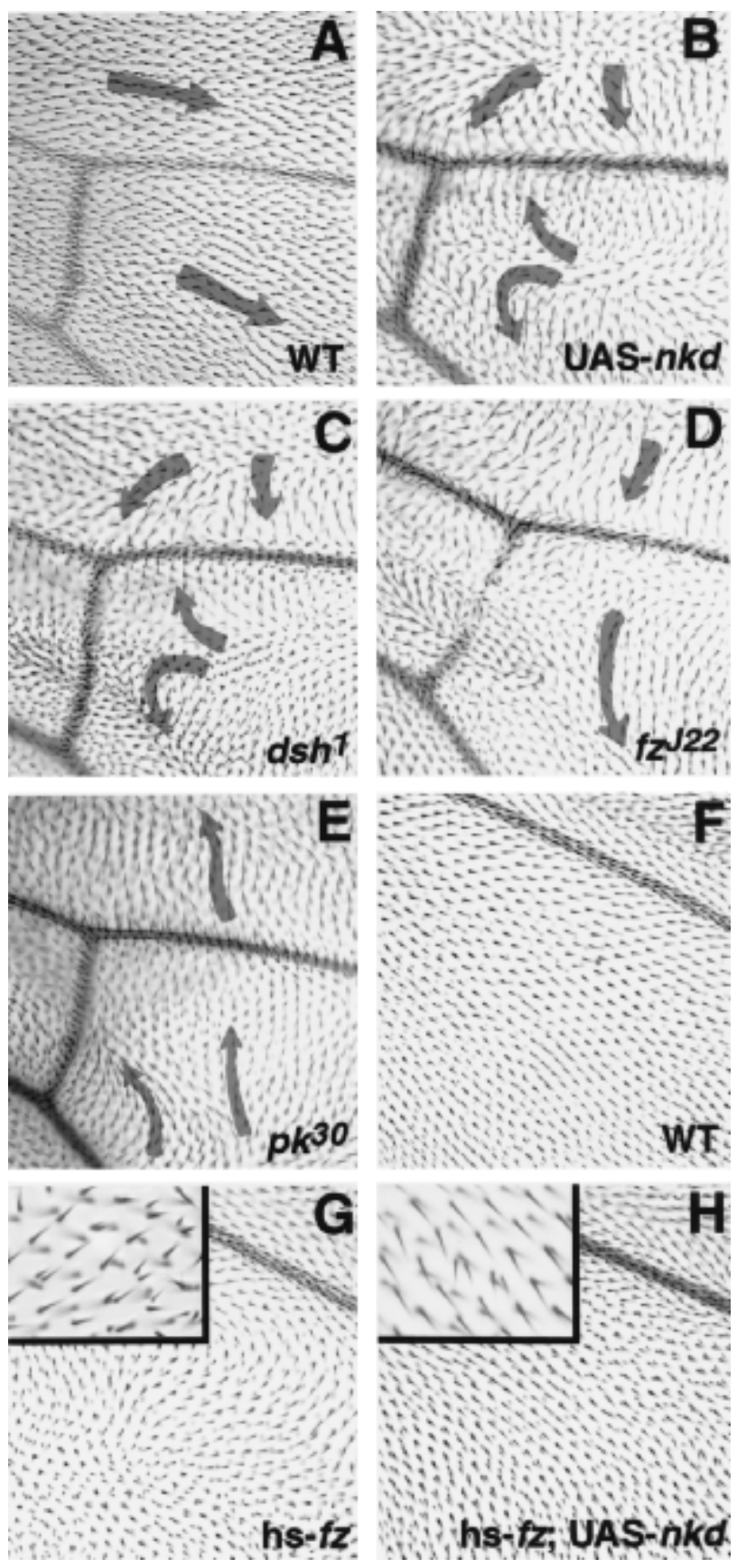

Figure 6. Effects of overproduced Nkd on planar cell polarity. (A) Wild-type (WT) wing pattern in region distal to posterior cross vein; same area shown in $B-E$. (B) Effect of overproduction of Nkd, which is similar to the phenotype shown in $C$, but different to the phenotypes shown in $D$ and $E .(C)$ Phenotype of loss of $d s h$ function ( $d s h^{1}$ allele). (D) Phenotype of loss of frizzled function $\left(f z^{I 22}\right.$ allele). (E) Phenotype of loss of prickle function ( $p k^{30}$ allele). Arrows indicate hair orientation. $(F)$ Wildtype wing pattern in the area shown in $G$ and $H$ : region posterior to vein 5. $(G)$ Phenotype of heat shock promoter-driven frizzled expression. $(H)$ Phenotype of heat shock promoter-driven $f z$ expression in the presence of UAS-nkd. Almost complete suppression of polarity defects by Nkd overexpression is observed. High magnification shows that $f z$ overexpression also induces double hair cells ( $G$ inset) and that their number decreases in the presence of $\mathrm{Nkd}$ ( $H$ inset). wg and $z w 3$ dsh mutants resemble $z w 3$ embryos (Siegfried et al. 1992, 1994; Peifer et al. 1994), whereas embryos doubly mutant for $n k d$ and either $w g$ (Bejsovec and Wieschaus 1993) or $d s h$ (this study) have a wg-like phenotype. In the absence of $\mathrm{Wg}, \mathrm{Zw} 3$ phosphorylates and causes degradation of Arm, whereas in cells receiving Wg signal, Zw3 activity is inactivated. If Zw3 function is eliminated by mutation, Arm becomes stable and stimulates target-gene transcription, regardless of upstream Wg or Dsh activities. In contrast, the removal of Nkd in wg or $d s h$ embryos does not lead to excess target-gene activity. Therefore, $\mathrm{Wg}$ does not act through Nkd-in the sense that it does through Zw3-to regulate en transcription and epidermal patterning. Taken together, our findings show that Nkd acts as a regulatory component that restrains $\mathrm{Wg}$ signal transduction.

The phenotypes of double-mutant embryos are consistent with the observation that the $n k d$ gene is itself regulated by Wg signaling (Zeng et al. 2000). The level of $n k d$ mRNA is markedly decreased in wg mutants (Zeng et al. 2000); so complete loss of $n k d$ (i.e., in a $n k d$ mutant) in a $w^{g}$ mutant would not be expected to dramatically alter the $w g$ phenotype. In contrast, zw3 transcripts are ubiquitous during embryonic stages (Bourouis et al. 1990; Siegfried et al. 1990), and no indication of Wg control of zw3 transcription has been found. Various signaling pathways are regulated in a negative feedback loop by components acting in the extracellular space, in the cytoplasm and/or in the nucleus (Perrimon and McMahon 1999; Freeman 2000). Those mechanisms elegantly buffer cellular responses against signal level fluctuations during pattern formation. Our results allow classification of Nkd as a novel intracellular negative feedback antagonist that acts cell-autonomously in the $\mathrm{Wg} / \mathrm{Wnt}$ pathway in a manner analogous to sprouty in the EGFR pathway, puckered in the JNK pathway, or daughters against $d p p$ in the TGF- $\beta / D p p$ pathway (Perrimon and McMahon 1999).

The biochemical and cell biological roles of Dsh in Wg and PCP signaling remain a mystery. Previous work showed that these two pathways employ Dsh in distinct ways, through different domains, to transduce signals (Axelrod et al. 1998; Boutros et al. 1998). Under certain circumstances Wg signaling can titrate Dsh from its function in planar cell polarity (Axelrod et al. 1998). We showed that aberrant $\mathrm{Nkd}$ production during times when Dsh is participating in PCP signaling results in polarity defects specific to loss of $d s h$ function, confirming that Nkd can affect the activity of endogenous Dsh. Polarized subcellular localization of PCP components appears to be essential for normal PCP signaling (Tomlinson and Struhl 1999; Usui et al. 1999). Control of Dsh subcellular localization has been implicated as a distinguishing feature in its two distinct roles in signal transduction: In a heterologous system, $\mathrm{Fz}$ is capable of recruiting Dsh to the plasma membrane, whereas DFz2, which is not involved in PCP signaling, is not (Axelrod et al. 1998). Recently, Dsh has been shown to regulate convergent extension movements during Xenopus gastrulation by modulating the frequency of filopodial exten- 
sions on adjacent faces of individual cells (Wallingford et al. 2000). Upon Wg signal activation, Dsh is phosphorylated, but the biochemical function of this phosphorylation is not known (Yanagawa et al. 1995). By directly interacting with Dsh, Nkd may sequester, degrade, or modify Dsh to block participation in PCP or Wg signaling. Future experiments will seek to elucidate the cellular and biochemical consequences of the interaction between Nkd and Dsh during Wg signaling.

That Nkd can act through Dsh has important implications for the dynamic control of $\mathrm{Wg} / \mathrm{Wnt}$ signaling. By acting upstream of the $\beta$-catenin degradation machinery, Nkd may determine how effectively a given dose of Wnt causes $\beta$-catenin accumulation and target-gene activation and thereby influence the sensitivity of a cell to a given amount or type of Wnt ligand. The kinetic and dynamic parameters of the feedback loop involving $\mathrm{Wg}$, Dsh, and Nkd may play key roles in controlling the duration and extent of signaling activity. Tight regulation of this feedback loop is clearly important for normal Drosophila embryonic development, and in various animals it may be subject to spatial and temporal adjustments during evolution or during disease progression. Future experiments will test how the interaction between Nkd and Dsh affects responses to Wnt signals during development and may provide insight into Wnt-associated tumor progression.

\section{Materials and methods}

nkd mutant eyes

$\mathrm{P}[$ FRT $80 B], h^{1} n k d^{7 H 16}$ or $h^{+} n k d^{7 E 89}$ or $h^{1} n k d^{9 G 33} / T M 3$ (Zeng et al. 2000) males were mated to y w; EGUF/EGUF; P[FRT 80B] cly+ $\mathrm{P}[$ GMR-hid]/TM2 females and eyes of nonbalancer progeny were photographed under a Leica M10 stereomicroscope. (EGUF) eyeless-Gal4/UAS-Flp chromosome; (cl) recessive cell lethal (Stowers and Schwarz 1999).

\section{Eye misexpression}

All UAS-transgenes were expressed using multiple repeats of the glass (gl) enhancer to drive GAL4 expression P[GMR-GAL4]. The transgenic lines used in the eye epistasis study were $\mathrm{P}[\mathrm{SeV}$ $w g], \mathrm{P}[G M R-G A L 4], \mathrm{P}[\mathrm{UAS}-d s h], \mathrm{P}\left[\mathrm{UAS}-\mathrm{arm}^{\text {s1o }}\right]$ (arm $^{\text {S10 }}$ encodes a constitutively active form of Arm protein with a 54amino acid deletion in the N-terminal domain; Pai et al. 1997), P[UAS-nkd 3-2], P[UAS-nkd 11], P[UAS-GPI-Dfz2], and P[UASlacZ]. All crosses were carried out at $29^{\circ} \mathrm{C}$. Adult offspring were prepared for scanning electron microscopy by treatment in a graded series of ethanol, followed by treatment in a graded series of hexamethyldisilazane. Dried samples were mounted on colloidal graphite and a 12-nm gold coat was applied with a Polaron Coating System. Samples were viewed with a Phillips 505 scanning electron microscope and photographed using Kodak 55 Instant Film.

\section{zw3/Gsk3ß expression in nkd mutants}

A 0-3.5 h collection of P[UAS-zw3]/P[UAS-zw3]; +/+; hs-GAL4, $n k d^{7 H 16} / \mathrm{TM} 3$ embryos was heat-shocked for $15 \mathrm{~min}$ at $37^{\circ} \mathrm{C}$ and allowed to recover overnight at $25^{\circ} \mathrm{C}$. The embryos were then dechorionated in $50 \%$ bleach. The cuticles were prepared as described by Willert et al. (1999) and were evaluated using phase contrast and darkfield microscopy. For the injection study, pCS2-XGsk3 $\beta$ DNA was linearized with NotI and singlestranded RNA transcripts were synthesized with Sp6 polymerase using Message Machine (Ambion). mRNA transcripts were visualized on a $1 \%$ gel and diluted to $1.0,0.5$, and $0.2 \mu \mathrm{g} / \mathrm{mL}$ in $\mathrm{ddH}_{2} \mathrm{O}$. Preblastoderm wild-type, $n k d^{7 E 89}$, or $n k d^{7 H 16}$ embryos were injected posteriorly with mRNA and allowed to complete embryogenesis. Cuticles were evaluated as stated above. Rescue tended to be greatest posteriorly at the site of injection.

\section{nkd misexpression clones}

To induce $n k d$ misexpression clones, $+/ Y ; \mathrm{P}[s e v-w g], \mathrm{P}[U A S$ nkd 3-2]; +/+ males were crossed with $y_{1}, W$, $\mathrm{P}[$ Actin $5 C>C D 2>G A L 4] ; \mathrm{P}[\mathrm{UAS}-G F P] ; \quad M K R S, \quad S b \mathrm{P}[\mathrm{hs}-F L P] /$ $T M 6 B, T b$ females. Second instar larvae were heat-shocked for 1 $\mathrm{h}$ in a $37^{\circ} \mathrm{C}$ water bath. For evaluation of clones in adult eyes, heads were dissected from the body and the eyes examined for GFP expression and suppression of bristle loss using a Zeiss Axioplan. Images were taken with a Princeton Micromax Digital Camera System. For evaluation of clones in pupal eyes, discs were dissected from pupae 30-36 h after pupal formation and fixed in $4 \%$ paraformaldehyde. Mouse monoclonal anti-Cut was used at a concentration of 1:100; rhodamine-conjugated goat anti-mouse antibody (Jackson Immunoresearch Labs) was used at a concentration of 1:200. Clones were evaluated and images taken using a Zeiss Axioskop and the MRC-1000 Laser Scanning Confocal Imaging System.

\section{Crosses for the dsh; nkd and arm; nkd double mutants}

The FLP-FRT system was used as described by Chou and Perrimon (1992) to generate the $d s h$ germ-line clones. Females of the genotypes y w dsh ${ }^{\mathrm{v} 26} \mathrm{FRT}^{101} / \mathrm{FM} 7 \mathrm{c} ;+/+$; nkd $\mathrm{d}^{716} \mathrm{ru} \mathrm{cu} \mathrm{ca/TM6B}$ or $y w d s h^{v 26} F R T^{101} / F M 7 c ;+/+; n k d^{7 E 89}$ ru cu ca/TM6B or y $w$ $d s h^{477} F^{2} T^{101} / F M 7 c ;+/+; n k d^{7 H 16}$ ru cu ca/TM6 were crossed with $W O V O^{D 1} V F R T^{101} / Y$; FLP $P^{38} / F L P^{38} ;+/+$ males. Early pupae were heat-shocked for $3 \mathrm{~h}$ in a $37^{\circ} \mathrm{C}$ water bath. y $w d s h$ $F R T^{101 / w}$ OVO $^{D 1}$ V FRT $T^{101}$; FLP $P^{38} /+$; nkd ru cu ca/+ females were selected and crossed with $n k d^{7 H 16}$ ru cu ca/TM3 or nkd $d^{7 E 89} \mathrm{ru}$ cu ca/TM3 males. Embryos were collected at 10 or $14 \mathrm{~h}$ and incubated at room temperature or $25^{\circ} \mathrm{C}$ until the first larvae hatched. The embryos were dechorionated in 50\% bleach and cuticles were prepared as described by Willert et al. (1999).

For the arm; nkd double mutant, females of the genotype $y$ $\operatorname{arm}^{Y D 35} / F M 7 ;$ + /+ were crossed with $+/ Y ; n k^{7 H 16}$ ru cu cal TM3 males. y arm ${ }^{Y D 35} /+; ; n k d^{7 H 16}$ ru cu ca/+ females were selected and mated to $+/ Y_{;} ; n k d^{7 H 16}$ ru cu ca/TM3 or $+/ Y_{;} ; n k d^{7 E 89}$ ru cu ca/TM3 males. Embryos were collected for 10 or $14 \mathrm{~h}$ and incubated at room temperature for $36 \mathrm{~h}$ to let the wild-type larvae crawl away. Cuticles of the unhatched embryos were prepared as indicated above.

\section{RNA interference}

The following oligonucleotides containing the $\mathrm{T} 7$ promoter were designed as described previously by Kennerdell and Carthew (1998) to amplify a fragment of 797 bp from the nkd cDNA: T7-GAAGAGCCATCACCACCAGTCG (sens) and T7GTATTGCAGCGTTGGCGTTGC (anti-sens). nkd dsRNA was synthesized from the PCR fragment using the MEGAscript in vitro transcription kit (Ambion) as recommended by the manufacturer. Injections in y $w$ flies and cuticle preparations were made as described by Willert et al. (1999). For injections in dsh embryos, y w ras $d s h^{75} F R T^{101} / F M 7 ;+/+$ females were 
crossed with $w$ OVO ${ }^{D 1} V F R T^{101} / Y$; FLP $P^{38} / F L P^{38}$ males and heatshocked as outlined above. y $\mathrm{W}$ ras $d s h^{75} \mathrm{FRT}^{101} / \mathrm{W} \mathrm{OVO}^{D 1} \mathrm{~V}$ $F R T^{101}$ females were selected from this cross and mated to $D f(1) J A 27 / F M 7 c, K r-G F P^{10}$ males. GFP-expressing embryos, which carry the rescue paternal X-chromosome, were eliminated between $19 \mathrm{~h}$ and $26 \mathrm{~h}$ after injection.

\section{Yeast two-hybrid assay}

The full-length $n k d$ coding sequence was inserted into the yeast expression vector pAS2-1 (Clontech). The coding sequences of $d s h$ (wt and mutants), zw3, arm, and the intracellular portion of $D f z 2$ (Dfz2CT, amino acids 607 to 694) were inserted into the yeast vector pACT2 (Clontech). The Dsh mutants D1 to D6 have been described by Yanagawa et al. (1995). The DEP, D6PDZ, and PDZ mutants correspond to amino acids 334-623, 167-338, and 248-338 of Dsh, respectively. Transformation of yeast strain PJ69-4A was performed using a variation of the lithium acetate method, and $\beta$-galactosidase activity (from the $\mathrm{LacZ}$ reporter gene) was assayed in a liquid-culture assay using O-nitrophenyl $\beta$-D-galactopyranoside as substrate (see Clontech protocols). Yeast-growth assay was performed using the $\mathrm{ADE}$ reporter gene and adenine minus medium.

\section{Immunoprecipitation}

COS-7 cells were transfected using calcium phosphate precipitation with pcDNA3.1B(-)/Myc-HisB vectors (Invitrogen) expressing Nkd and Dsh proteins tagged with the myc epitope at the $\mathrm{C}$ terminus. After $40 \mathrm{~h}$, cells were lysed in TNN75 buffer $(25$ $\mathrm{mM}$ Tris- $\mathrm{HCl}$ at $\mathrm{pH} 8.0,75 \mathrm{mM} \mathrm{NaCl}, 0.5 \%$ IGEPAL CA-630, $1 \mathrm{mM}$ DTT, $1 \mathrm{mM}$ Pefabloc SC, antiprotease cocktail). Immunoprecipitation was carried out in TNN75-10\% glycerol using rabbit anti-Dsh antibody (affinity-purified) and protein A Sepharose-4B beads. Immunoprecipitate was washed in TNN75 buffer and proteins were eluted with SDS-loading buffer and then run on SDS protein gels. Western blot was performed using anti-c-Myc antibody and enhanced chemiluminescence (Pierce).

\section{GST pull-down assay}

The coding sequence of full-length $n k d$ was inserted into the pGEX-4T-1 vector (Pharmacia Biotech). Plasmid expressing GST-Dsh has been described by Willert et al. (1997). Bacterial lysates containing the GST fusion proteins were prepared as described by Pharmacia Biotech, except MTPBS buffer (150 mM $\mathrm{NaCl}, 12.5 \mathrm{mM} \mathrm{Na}_{2} \mathrm{HPO}_{4}, 2.5 \mathrm{mM} \mathrm{KH}_{2} \mathrm{PO}_{4}, 1 \mathrm{mM}$ Pefabloc SC, $5 \mathrm{mM}$ DTT, and antiprotease cocktail) was used instead of PBS. The lysates were bound to glutathione-sepharose 4B beads and the beads resuspended in DT80 buffer $(20 \mathrm{mM}$ Tris- $\mathrm{HCl}$ at $\mathrm{pH} 8$, $80 \mathrm{mM} \mathrm{KCl}, 0.25 \%$ Triton X-100, $1 \mathrm{mM}$ Pefabloc SC, $1 \mathrm{mM}$ DTT). The beads were then incubated with $\left[{ }^{35} S\right]$ methioninelabeled Nkd or Dsh (full length or mutant), which were produced using the TNT T7 Coupled Reticulocyte Lysate System (Promega) from pBluescript IIKS $(+)$ plasmids. The beads were washed with DT300 buffer (20 mM Tris- $\mathrm{HCl}$ at $\mathrm{pH} 8,300 \mathrm{mM}$ $\mathrm{KCl}, 0.25 \%$ Triton X-100) and incubated with SDS-loading buffer to elute the proteins. Samples were run on SDS protein gels.

\section{Planar cell polarity assay}

To assay effects of overexpression on planar polarity, white prepupae were selected and placed in plastic vials, aged for $24 \mathrm{~h}$ at $25^{\circ} \mathrm{C}$, immersed for $2 \mathrm{~h}$ in a $37^{\circ} \mathrm{C}$ water bath and then allowed to develop at $25^{\circ} \mathrm{C}$. Wings were mounted in Euparal. The geno- types were UAS-nkd 3-2/+; hs-GAL4/+ (Fig. 6B), hs-fz30.9/+ (Fig. 6G), UAS-nkd 3-2/hs-fz30.9; hs-GAL4, nk $d^{7 H 16} /+($ Fig. 6H); and $w, d s h^{1} / Y$; UAS-nkd 3-2/+; hs-GAL4, nkd $d^{7 H 16}$ (data not shown).

\section{Acknowledgments}

We thank P. Klein for the pCS2-XGSK3 $\beta$ construct; E. Rulifson for the FLP-out/GAL4 line; K. Willert for anti-Dsh antibody; the Developmental Studies Hybridoma Bank for anti-Cut antibody; S. Stowers and T. Schwarz for EGUF/hid stocks; the Bloomington Stock Center for the ${ } v O^{D 1}$ fly stock; and the Axelrod, Nusse, and Scott labs for reagents, advice, and encouragement. R.R. was supported by the Association pour la Recherche sur le Cancer and by the Human Frontier Science Program. J.A.M. was supported by an NIH postdoctoral fellowship and the Howard Hughes Medical Institute (HHMI). K.A.W. was supported by a K-08 award from the NIH. J.D.A. was supported in part by DRS 16 of the Cancer Research Fund of the Damon Runyon-Walter Winchell Foundation, and by the HHMI. K.C. was supported by a grant from the NIH (RO1 GM59846) and by the HHMI. R.N. and M.P.S. are investigators of the HHMI.

The publication costs of this article were defrayed in part by payment of page charges. This article must therefore be hereby marked "advertisement" in accordance with 18 USC section 1734 solely to indicate this fact.

\section{References}

Aberle, H., Bauer, A., Stappert, J., Kispert, A., and Kemler, R. 1997. $\beta$-catenin is a target for the ubiquitin-proteasome pathway. EMBO I. 16: 3797-3804.

Ahmed, Y., Hayashi, S., Levine, A., and Wieschaus, E. 1998. Regulation of armadillo by a Drosophila APC inhibits neuronal apoptosis during retinal development. Cell 93: 11711182.

Axelrod, J.D., Miller, J.R., Shulman, J.M., Moon, R.T., and Perrimon, N. 1998. Differential recruitment of Dishevelled provides signaling specificity in the planar cell polarity and Wingless signaling pathways. Genes \& Dev. 12: 2610-2622.

Bejsovec, A. and Wieschaus, E. 1993. Segment polarity gene interactions modulate epidermal patterning in Drosophila embryos. Development 119: 501-517.

Bhanot, P., Brink, M., Samos, C.H., Hsieh, J.C., Wang, Y., Macke, J.P., Andrew, D., Nathans, J., and Nusse, R. 1996. A new member of the frizzled family from Drosophila functions as a Wingless receptor. Nature 382: 225-230.

Bourouis, M., Moore, P., Ruel, L., Grau, Y., Heitzler, P., and Simpson, P. 1990. An early embryonic product of the gene shaggy encodes a serine/threonine protein kinase related to the CDC28/cdc2 + subfamily. EMBO J. 9: 2877-2884.

Boutros, M. and Mlodzik, M. 1999. Dishevelled: At the crossroads of divergent intracellular signaling pathways. Mech. Dev. 83: 27-37.

Boutros, M., Paricio, N., Strutt, D.I., and Mlodzik, M. 1998. Dishevelled activates JNK and discriminates between JNK pathways in planar polarity and wingless signaling. Cell 94: 109-118.

Brunner, E., Peter, O., Schweizer, L., and Basler, K. 1997. pangolin encodes a Lef- 1 homologue that acts downstream of Armadillo to transduce the Wingless signal in Drosophila. Nature 385: 829-833.

Cadigan, K.M. and Nusse, R. 1996. wingless signaling in the Drosophila eye and embryonic epidermis. Development 122: 
2801-2812.

. 1997. Wnt signaling: A common theme in animal development. Genes \& Dev. 11: 3286-3305.

Cadigan, K.M., Grossniklaus, U., and Gehring, W.J. 1994. Localized expression of sloppy paired protein maintains the polarity of Drosophila parasegments. Genes \& Dev. 8: 899913.

Cadigan, K.M., Fish, M.P., Rulifson, E.J., and Nusse, R. 1998. Wingless repression of Drosophila frizzled 2 expression shapes the Wingless morphogen gradient in the wing. Cell 93: 767-777.

Chou, T.B. and Perrimon, N. 1992. Use of a yeast site-specific recombinase to produce female germline chimeras in Drosophila. Genetics 131: 643-653.

DiNardo, S., Sher, E., Heemskerk-Jorgens, J., Kassis, J., and O'Farrell, P. 1988. Two-tiered regulation of spatially patterned engrailed gene expression during Drosophila embryogenesis. Nature 332: 604-609.

Dougan, S. and DiNardo, S. 1992. Drosophila wingless generates cell type diversity among engrailed expressing cells. $\mathrm{Na}$ ture 360: 347-350.

Freeman, M. 2000. Feedback control of intercellular signalling in development. Nature 408: 313-319.

Gubb, D. and Garcia-Bellido, A. 1982. A genetic analysis of the determination of cuticular polarity during development in Drosophila melanogaster. J. Embryol. Exp. Morphol. 68: 3757.

Hamada, F., Tomoyasu, Y., Takatsu, Y., Nakamura, M., Nagai, S., Suzuki, A., Fujita, F., Shibuya, H., Toyoshima, K., Ueno, N., et al. 1999. Negative regulation of Wingless signaling by D-axin, a Drosophila homolog of axin. Science 283: 17391742.

Ikeda, S., Kishida, S., Yamamoto, H., Murai, H., Koyama, S., and Kikuchi, A. 1998. Axin, a negative regulator of the Wnt signaling pathway, forms a complex with GSK-3beta and beta-

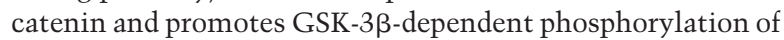
ß-catenin. EMBO J. 17: 1371-1384.

Ingham, P.W., Taylor, A.M., and Nakano, Y. 1991. Role of the Drosophila patched gene in positional signalling. Nature 353: $184-187$.

Jürgens, G., Wieschaus, E., Nüsslein-Volhard, C., and Kluding, H. 1984. Mutations affecting the pattern of the larval cuticle in Drosophila melanogaster. II. Zygotic loci on the third chromosome. Wilhelm Roux Arch. Dev. Biol. 193: 283-295.

Kennerdell, J.R. and Carthew, R.W. 1998. Use of dsRNA-mediated genetic interference to demonstrate that frizzled and frizzled 2 act in the wingless pathway. Cell 95: 1017-1026.

Klingensmith, J. and Nusse, R. 1994. Signaling by wingless in Drosophila. Dev. Biol. 166: 396-414.

Klingensmith, J., Nusse, R., and Perrimon, N. 1994. The Drosophila segment polarity gene dishevelled encodes a novel protein required for response to the wingless signal. Genes \& Dev. 8: 118-130.

Krasnow, R.E. and Adler, P.N. 1994. A single frizzled protein has a dual function in tissue polarity. Development 120: 1883-1893.

Lee, J.J., von Kessler, D.P., Parks, S., and Beachy, P.A. 1992. Secretion and localized transcription suggest a role in positional signaling for products of the segmentation gene hedgehog. Cell 71: 33-50.

Li, L., Yuan, H., Weaver, C.D., Mao, J., Farr III, G.H., Sussman, D.J., Jonkers, J., Kimelman, D., and Wu, D. 1999. Axin and Fratl interact with dvl and GSK, bridging Dvl to GSK in Wnt- mediated regulation of LEF-1. EMBO T. 18: 4233-4240.

Martinez Arias, A., Baker, N.E., and Ingham, P.W. 1988. Role of segment polarity genes in the definition and maintenance of cell states in the Drosophila embryo. Development 103: 157-170.

McCartney, B.M., Dierick, H.A., Kirkpatrick, C., Moline, M.M., Baas, A., Peifer, M., and Bejsovec, A. 1999. Drosophila APC2 is a cytoskeletally-associated protein that regulates wingless signaling in the embryonic epidermis. J. Cell. Biol. 146: 1303-1318.

Moline, M.M., Southern, C., and Bejsovec, A. 1999. Directionality of Wingless protein transport influences epidermal patterning in the Drosophila embryo. Development 126: 43754384.

Noordermeer, J., Johnston, P., Rijsewijk, F., Nusse, R., and Lawrence, P.A. 1992. The consequences of ubiquitous expression of the wingless gene in the Drosophila embryo. Development 116: 711-719.

Noordermeer, J., Klingensmith, J., Perrimon, N., and Nusse, R. 1994. dishevelled and armadillo act in the wingless signalling pathway in Drosophila. Nature 367: 80-83.

Nusse, R. and Varmus, H.E. 1982. Many tumors induced by the mouse mammary tumor virus contain a provirus integrated in the same region of the host genome. Cell 31: 99-109.

O'Keefe, L., Dougan, S.T., Gabay, L., Raz, E., Shilo, B.Z., and DiNardo, S. 1997. Spitz and Wingless, emanating from distinct borders, cooperate to establish cell fate across the Engrailed domain in the Drosophila epidermis. Development 124: 4837-4845.

Pai, L.M., Orsulic, S., Bejsovec, A., and Peifer, M. 1997. Negative regulation of Armadillo, a Wingless effector in Drosophila. Development 124: 2255-2266.

Peifer, M. and Wieschaus, E. 1990. The segment polarity gene armadillo encodes a functionally modular protein that is the Drosophila homolog of human plakoglobin. Cell 63: 11671176.

Peifer, M., Sweeton, D., Casey, M., and Wieschaus, E. 1994. wingless signal and Zeste-white 3 kinase trigger opposing changes in the intracellular distribution of Armadillo. Development 120: 369-380.

Perrimon, N. and Mahowald, A.P. 1987. Multiple functions of segment polarity genes in Drosophila. Dev. Biol. 119: 587600.

Perrimon, N. and Smouse, D. 1989. Multiple functions of a Drosophila homeotic gene, zeste-white 3, during segmentation and neurogenesis. Dev. Biol. 135: 287-305.

Perrimon, N. and McMahon, A.P. 1999. Negative feedback mechanisms and their roles during pattern formation. Cell 97: 13-16.

Peters, J.M., McKay, R.M., McKay, J.P., and Graff, J.M. 1999. Casein kinase I transduces Wnt signals. Nature 401: 345350.

Polakis, P. 2000. Wnt signaling and cancer. Genes \& Dev. 14: 1837-1851.

Shulman, J.M., Perrimon, N., and Axelrod, J.D. 1998. Frizzled signaling and the developmental control of cell polarity. Trends Genet. 14: 452-458.

Siegfried, E., Perkins, L.A., Capaci, T.M., and Perrimon, N. 1990. Putative protein kinase product of the Drosophila segment-polarity gene zeste-white3. Nature 345: 825-829.

Siegfried, E., Chou, T.B., and Perrimon, N. 1992. wingless signaling acts through zeste-white 3 , the Drosophila homolog of glycogen synthase kinase-3, to regulate engrailed and establish cell fate. Cell 71: 1167-1179.

Siegfried, E., Wilder, E.L., and Perrimon, N. 1994. Components of wingless signalling in Drosophila. Nature 367: 76-80.

Stowers, R.S. and Schwarz, T.L. 1999. A genetic method for generating Drosophila eyes composed exclusively of mitotic clones of a single genotype. Genetics 152: 1631-1639. 
Szuts, D., Freeman, M., and Bienz, M. 1997. Antagonism between EGFR and Wingless signalling in the larval cuticle of Drosophila. Development 124: 3209-3219.

Theisen, H., Purcell, J., Bennett, M., Kansagara, D., Syed, A., and Marsh, J.L. 1994. dishevelled is required during wingless signaling to establish both cell polarity and cell identity. Development 120: 347-360.

Tomlinson, A. and Struhl, G. 1999. Decoding vectorial information from a gradient: Sequential roles of the receptors Frizzled and Notch in establishing planar polarity in the Drosophila eye. Development 126: 5725-5738.

Usui, T., Shima, Y., Shimada, Y., Hirano, S., Burgess, R.W., Schwarz, T.L., Takeichi, M., and Uemura, T. 1999. Flamingo, a seven-pass transmembrane cadherin, regulates planar cell polarity under the control of Frizzled. Cell 98: 585595.

Van de Wetering, M., Cavallo, R., Dooijes, D., van Beest, M., van Es, J., Loureiro, J., Ypma, A., Hursh, D., Jones, T., Bejsovec, A., et al. 1997. Armadillo coactivates transcription driven by the product of the Drosophila segment polarity gene dTCF. Cell 88: 789-799.

Van den Heuvel, M., Klingensmith, J., Perrimon, N., and Nusse, R. 1993. Cell patterning in the Drosophila segment: Engrailed and wingless antigen distributions in segment polarity mutant embryos. Dev. Suppl. 105-114.

Vincent, J.P. and O'Farrell, P.H. 1992. The state of engrailed expression is not clonally transmitted during early Drosophila development. Cell 68: 923-931.

Wallingford, J.B., Rowning, B.A., Vogeli, K.M., Rothbacher, U., Fraser, S.E., and Harland, R.M. 2000. Dishevelled controls cell polarity during Xenopus gastrulation. Nature 405: 8185.

Willert, K., Brink, M., Wodarz, A., Varmus, H., and Nusse, R. 1997. Casein kinase 2 associates with and phosphorylates dishevelled. EMBO J. 16: 3089-3096.

Willert, K., Logan, C.Y., Arora, A., Fish, M., and Nusse, R. 1999. A Drosophila Axin homolog, Daxin, inhibits Wnt signaling. Development 126: 4165-4173.

Yanagawa, S., van Leeuwen, F., Wodarz, A., Klingensmith, J., and Nusse, R. 1995. The dishevelled protein is modified by wingless signaling in Drosophila. Genes \& Dev. 9: 10871097.

Yost, C., Torres, M., Miller, J.R., Huang, E., Kimelman, D., and Moon, R.T. 1996. The axis-inducing activity, stability, and subcellular distribution of $\beta$-catenin is regulated in Xenopus embryos by glycogen synthase kinase 3. Genes \& Dev. 10: 1443-1454.

Zeng, W., Wharton, Jr., K.A., Mack, J.A., Wang, K., Gadbaw, M., Suyama, K., Klein, P.S., and Scott, M.P. 2000. naked cuticle encodes an inducible antagonist of Wnt signalling. Nature 403: 789-795. 


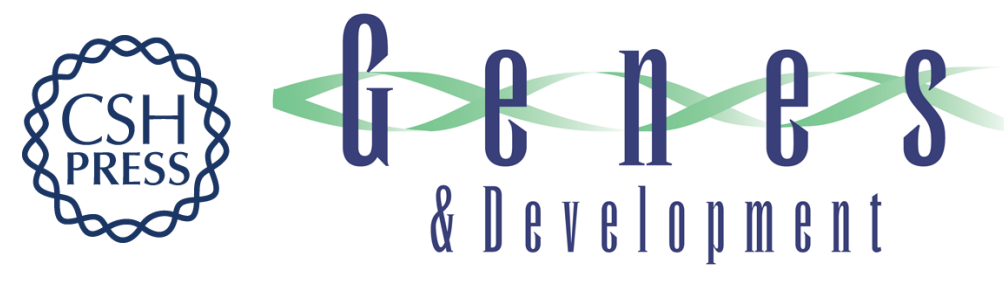

\section{naked cuticle targets dishevelled to antagonize Wnt signal transduction}

Raphaël Rousset, Judith A. Mack, Keith A. Wharton, Jr., et al.

Genes Dev. 2001, 15:

Access the most recent version at doi:10.1101/gad.869201

References This article cites 60 articles, 28 of which can be accessed free at: http://genesdev.cshlp.org/content/15/6/658.full.htmI\#ref-list-1

License

Email Alerting

Receive free email alerts when new articles cite this article - sign up in the box at the top Service right corner of the article or click here.

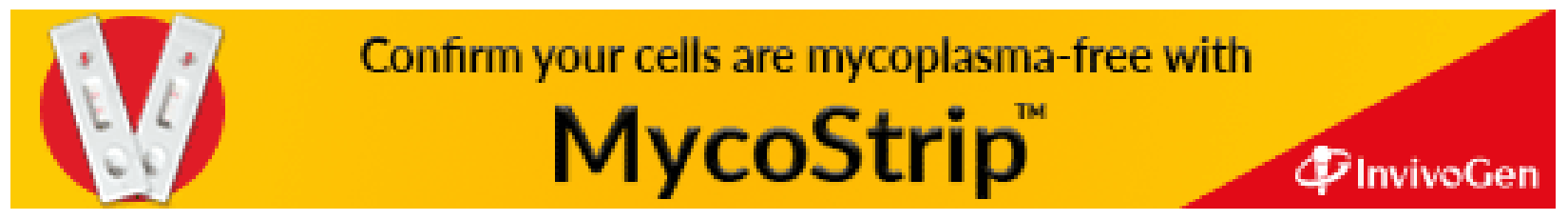

